\title{
ARTí́CULO
}

\section{Identificación de zonas propicias para el desarrollo larval de la macarela del Pacífico (Scomber japonicus) en la porción sur de la Corriente de California}

Identification of suitable areas for the larval development of the Pacific mackerel (Scomber japonicus) in the southern portion of the California Current

\author{
Eduardo Anaya-Godínez ${ }^{1 *}$, René Funes-Rodríguez ${ }^{1}$, Alejandro T. \\ Hinojosa-Medina ${ }^{1}$, Adrián F. González-Acosta ${ }^{1}$, José L. \\ Ortíz-Galindo ${ }^{1}$, Eduardo González-Rodríguez ${ }^{2}$
} y Mauricio F. Landaeta ${ }^{3}$

\begin{abstract}
'Instituto Politécnico Nacional, Centro Interdisciplinario de Ciencias Marinas, Av. IPN s/n, Col. Playa Palo de Santa Rita, C.P. 23096. La Paz, Baja California Sur, México. "eanayag1400@alumno.ipn.mx

${ }^{2}$ Centro de Investigación Científica y de Educación Superior de Ensenada, Unidad La Paz, Calle Miraflores 334, Fraccionamiento Bella Vista, C.P. 23050, La Paz, Baja California Sur, México

${ }^{3}$ Laboratorio de Ictioplancton (LABITI), Facultad de Ciencias del Mar y de Recursos Naturales, Universidad de Valparaíso, Av. Borgoño 16344, Reñaca, Viña del Mar, Chile

Abstract.- Larval size is considered as an important variable to understand the changes in larval development and identification of a suitable environment for larval growth and survivor. Larvae of the Pacific mackerel (Scomber japonicus) have a slow growth during the early stages of development (6-8 $\mathrm{mm} \mathrm{SL);} \mathrm{subsequently,} \mathrm{the} \mathrm{growth} \mathrm{rate} \mathrm{increases,} \mathrm{but} \mathrm{the} \mathrm{development} \mathrm{depends} \mathrm{mainly}$ on the temperature and food. The aim of this study was to identify the areas and seasons favorable for larval development of $S$. japonicus, in relation to the sea surface temperature and the zooplankton biomass, off the west coast of the Baja California Peninsula $\left(\sim 25^{\circ}-32^{\circ} \mathrm{N}\right)$ from 2006 to 2010 . Larvae of the Pacific mackerel showed the greatest amplitude in their spatial distribution during spring, but with greater restricted abundance compared to Punta Eugenia $\left(\sim 28^{\circ} \mathrm{N}\right)$ during summer. Changes in its distribution were influenced by geostrophic flow and thermal variability. Linear regression analysis allowed to identify areas favorable for larvae development, with a lower development in spring, related to a narrow range of temperatures and levels of comparatively low zooplankton biomass. In contrast, during summer the largest increase in body height $(\beta)$ was related to a wide range of temperatures, while its low variability (standard error) coincided with high levels of zooplankton biomass. It is considered that thermal conditions and food availability, favor an optimum larval development, particularly during the summer season.
\end{abstract}

Key words: Pacific mackerel, fish larvae, isometric growth, suitable areas, California Current

Resumen.- El tamaño larval es una variable importante para entender los cambios en el desarrollo y la identificación de un entorno adecuado para el crecimiento y la supervivencia larval. Las larvas de la macarela del Pacífico (Scomber japonicus) presentan un lento crecimiento durante las primeras fases del desarrollo (6-8 $\mathrm{mm}$ de LE); posteriormente, la velocidad de crecimiento aumenta, pero el desarrollo depende principalmente de la temperatura y del alimento. El objetivo del presente trabajo fue identificar las zonas y temporadas propicias para el desarrollo larval de S. japonicus, en relación a la temperatura superficial del mar y la biomasa del zooplancton, frente a la costa occidental de la Península de Baja California ( $\left.25-32^{\circ} \mathrm{N}\right)$ del 2006 al 2010. Las larvas de la macarela del Pacífico mostraron la mayor amplitud en su distribución espacial en primavera, pero con mayor abundancia restringida frente a Punta Eugenia $\left(\sim 28^{\circ} \mathrm{N}\right)$ durante el verano. Los cambios en su distribución, estuvieron bajo la influencia del flujo geostrófico y la variabilidad térmica. El análisis de regresión lineal permitió identificar zonas propicias para el desarrollo de larvas, con un menor desarrollo en primavera, relacionado con un intervalo estrecho de temperaturas y niveles de biomasas del zooplancton comparativamente bajos. En contraste, durante el verano el mayor incremento en la altura del cuerpo ( $\beta$ ) fue relacionado con un amplio intervalo de temperaturas, mientras que su baja variabilidad (error estándar) coincidió con altos niveles de biomasa del zooplancton. Se considera que las condiciones térmicas y de disponibilidad de alimento favorecen un óptimo desarrollo larval particularmente en la época de verano.

Palabras clave: Macarela del Pacífico, larvas de peces, crecimiento isométrico, zonas propicias, Corriente de California 


\section{INTRODUCCIÓN}

El período larval de muchas especies de peces es considerado como un 'periodo crítico', debido a que quedan expuestos a una alta mortalidad y vulnerabilidad a la depredación e inanición (Li \& Mathias 1987, Stouthamer \& Bain 2012). Este periodo comienza cuando la larva es capaz de capturar organismos y se extiende, hasta presentar el tamaño y edad marcada por la formación del esqueleto axial. De forma simultánea, el desarrollo de las aletas con radios y espinas mejoran la habilidad para capturar el alimento (Stouthamer \& Bain 2012). Durante el periodo crítico, las larvas son susceptibles a requerimientos ambientales y necesidades de hábitat específicas (e.g., temperatura, refugio, corrientes y disponibilidad del alimento), que son claramente diferentes a los de juveniles y adultos (Werner 2002).

Un 'hábitat apropiado' para las primeras fases de vida de los peces, es difícil de concebir; no obstante, garantiza un mayor crecimiento y supervivencia larval, es por ello que las tallas son especialmente sensibles a la variabilidad ambiental y, en consecuencia, pudieran ser un indicador de la calidad del hábitat (Hare \& Cowen 1997, Werner 2002).

La macarela del Pacífico, Scomber japonicus Houttuyn, 1782, es una especie pelágica costera que se distribuye en el Pacífico nororiental, desde el sureste de Alaska hasta Bahía de Banderas (Moser et al. 1993). Las larvas de esta especie se pueden encontrar durante todo el año, con mayor abundancia en primavera y verano, en la parte norte y centro de la costa occidental de la Península de Baja California (COPBC), pero con menor abundancia en otoño (Kramer 1969, Moser et al. 1993). En esta región, se ha sugerido la existencia de 2 stocks de macarela con base en su madurez gonádica (Gluyas-Millán \& Quiñónez-Velázquez 1996). El primero se localiza frente a Bahía Magdalena $\left(24^{\circ} \mathrm{N}\right)$, cuya madurez gonadal sugiere un máximo desove durante invierno (Arcos-Huitrón \& TorresVillegas 1990) y en asociación con una alta abundancia larval (Moser et al. 1993). El segundo, se ubica en Bahía San Sebastián Vizcaíno $\left(28^{\circ} \mathrm{N}\right)$, el cual se extiende hacia el norte durante primavera (Gluyas-Millán \& Quiñónez-Velázquez 1996) y cuya presencia coincide con una menor abundancia larval (Moser et al. 1993); sin embargo, durante la época de verano el stock se contrae y al parecer tiene lugar un máximo del desove y alta abundancia de larvas, particularmente frente a Punta Eugenia $\left(\sim 28^{\circ} \mathrm{N}\right)$ (Moser et al. 1993, Gluyas-Millán 1994).

Las larvas de $S$. japonicus, al igual que las de otras especies de hábitos similares, fluctúan en el tiempo y zonas de desove a lo largo del borde exterior de la plataforma continental entre California y México (Lo et al. 2010, Weber \& McClatchie
2012). Durante los eventos El Niño, ocurre un incremento en la supervivencia de las larvas asociado a una reducción en el transporte de la Corriente de California (CC) hacia el sur. Por el contrario, en años La Niña, las tasas de supervivencia pueden ser comparativamente bajas, pero con un aumento en el transporte de la CC y en la biomasa de plancton (Sinclair et al. 1985). No obstante, las densidades larvales de esta especie han disminuido desde mediados de la década de los 80 “s y hasta el 2000, aunque se considera que esta tendencia puede continuar (Hsieh et al. 2005, Lo et al. 2010).

La variabilidad de la abundancia de larvas de macarela puede estar asociada con la biomasa del zooplancton (BZ), los cambios de temperatura, el flujo geostrófico y la latitud geográfica (Sinclair et al. 1985, Weber \& McClatchie 2012). La BZ pudiera no reflejar el tamaño y la calidad del alimento necesario asociado a las tasas de supervivencia, la estimación del volumen de zooplancton está altamente correlacionada con la biomasa del microplancton (Sinclair et al. 1985). La temperatura, determina no solo la duración del crecimiento, también cambia el patrón de desarrollo (Pörtner et al. 2010). A menor temperatura, la eclosión se retrasa y el desarrollo larval se prolonga, pero a temperaturas más altas, la eclosión, la natación de las larvas y el inicio de la alimentación exógena ocurre en un tamaño de cuerpo claramente más pequeño (Pörtner et al. 2010).

El transporte oceánico influye de forma importante durante el desarrollo inicial de los peces, debido al desplazamiento de los organismos desde, o hacia las zonas propicias para su supervivencia (Hare \& Cowen 1997). No obstante, las estrategias de reproducción de muchas especies marinas están adaptadas para ajustarse a las corrientes predominantes, y ser transportadas hacia las zonas de crianza, generalmente en aguas poco profundas o costeras (Parrish et al. 1981, Norcross \& Shaw 1984, Hare \& Cowen 1997); o bien, para formar parte de las comunidades biológicas que se desarrollan en algunos remolinos, debido a que ofrecen condiciones de retención de organismos planctónicos (Asch \& Checkley 2013). De igual forma, el éxito de una población dependerá en gran parte de la habilidad de las larvas para permanecer agregadas durante los primeros meses de vida (Sinclair 1988). Así como también, la localidad y extensión de áreas de retención geográficamente estables, que pueden determinar el número y localización de los stocks y sitios de desove (Iles \& Sinclair 1982).

Existen diversos criterios para analizar el desarrollo larval de los peces, como los histológicos (O'Connell 1976, Theilacker 1978, Ferron \& Leggett 1994), el factor de condición (Ehrlich et al. 1976, Westernhagen \& Rosenthal 1981), cambios 
bioquímicos y la relación ARN/ADN (Buckley 1984, Clemmesen \& Doan 1996, Chícharo 1998). Sin embargo, los criterios morfométricos reflejan, al igual que las técnicas anteriores, la condición de las larvas durante el desarrollo, y pueden emplearse fácilmente sin una preparación especial de los especímenes, aunado a su bajo costo y corto tiempo de procesamiento (Theilacker 1978, Catalán-Alemani 2003).

El crecimiento de las larvas de peces, sea alométrico o isométrico, es una estrategia que resulta de una forma específica del cuerpo, que puede variar dependiendo del ambiente en el que se desarrollen (Froese 1990). Por su parte, la variabilidad en el incremento de la altura del cuerpo (AC) con respecto de la longitud estándar (LE), puede utilizarse como un indicador de la condición nutricional de las larvas después de la absorción del saco vitelino (Theilacker 1978, Ehrlich et al. 1976, Yúfera et al. 1993) y consecuentemente, de la condición durante el desarrollo (Hunter \& Kimbrell 1980). Las larvas de $S$. japonicus presentan un lento crecimiento durante los primeros 6 a 8 mm de LE; después de esta talla, su crecimiento es más rápido y alcanza su completa transformación entre 2 y 3 semanas ( $\sim 15 \mathrm{~mm}$ de LE). Este desarrollo depende tanto del alimento como de la temperatura en un intervalo relativamente constante $\left(14-22^{\circ} \mathrm{C}\right.$ ) en el cual estas ocurren (Kramer 1960, Hunter \& Kimbrell 1980, Esqueda-Escárcega 1995, Sassa \& Tsukamoto 2010).
En este trabajo se analiza la proporción entre la altura del cuerpo y la longitud estándar de las larvas de la macarela del Pacífico (Scomber japonicus), en relación a la temperatura superficial del mar (TSM) y la biomasa del zooplancton (BZ) con objetivo de identificar las zonas y épocas propicias para un mejor desarrollo en el área frente a la COPBC. De forma simultánea se utiliza la TSM y el flujo geostrófico para identificar estructuras oceanográficas de mesoescala, con la finalidad de conocer su influencia en el hábitat preferencial de las larvas.

\section{Materiales y MÉTODOS}

\section{Área de estudio}

Se ubica frente a la costa occidental de la Península de Baja California, desde los $\sim 25^{\circ} \mathrm{N}$, hasta los $\sim 32^{\circ} \mathrm{N}$ y desde los $\sim 112^{\circ} \mathrm{W}$ a $\sim 119^{\circ} \mathrm{W}$ (Fig. 1). El área comprende desde la zona marina que se localiza entre Ensenada $\left(\sim 32^{\circ} \mathrm{N}\right)$, Baja California, hasta el sur del Golfo de Ulloa $\left(\sim 25^{\circ} \mathrm{N}\right)$, Baja California Sur. En el área se distinguen dos regiones geográficamente separadas. La norte ubicada entre Ensenada y norte de Punta Eugenia $\left(\sim 32^{\circ}-28^{\circ} \mathrm{N}\right)$ que se caracteriza por la dominancia de aguas subárticas durante todo el año, y la provincia al sur de Punta Eugenia $\left(\sim 28^{\circ}-25^{\circ} \mathrm{N}\right)$ caracterizada por la alternancia entre el dominio subártico (invierno-primavera) y tropical-subtropical (verano y otoño) (Hickey 1979, Lynn \& Simpson 1987, Durazo et al. 2010).

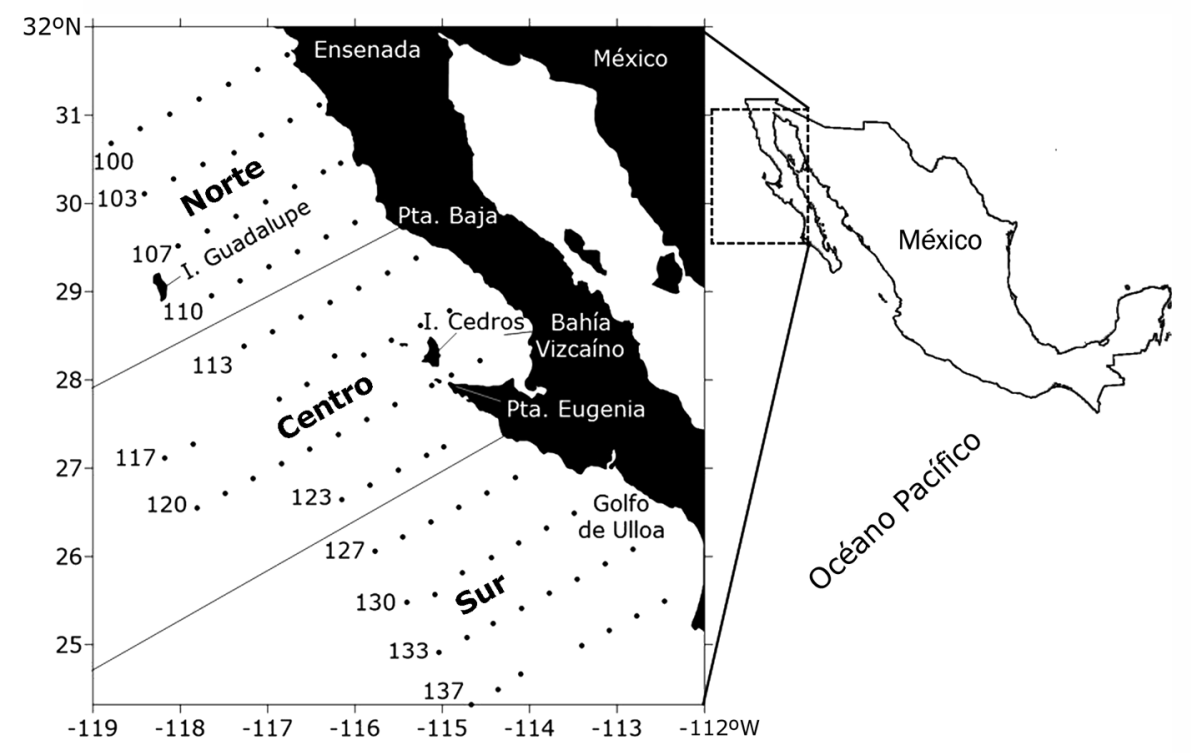

Figura 1. Localización del área de estudio y de las estaciones de muestreo del programa IM ECOCAL, en la costa occidental de la península de Baja California. Las líneas dentro del mapa representan el límite de las zonas divididas en el área de estudio (texto en negritas): zona norte (líneas 100 a 110), zona centro (líneas 113 a 123) y zona sur (líneas 127 a 137) / Location of the study area and sampling stations IMECOCAL program, on the west coast of the peninsula of Baja California. The lines within the map represent the boundary of the divided zones the study area (bold text): north zone (lines 100-110), central zone (lines 113-123) and south zone (lines 127-137) 
El área de estudio se caracteriza por presentar surgencias costeras durante todo el año en la región norte, mientras que en la región sur solo son evidentes en primavera y verano (Lynn \& Simpson 1987, Durazo et al. 2010). Durante la primavera y verano, la CC fluye hacia el sur y en otoño su flujo persiste, aunque separado de la costa; en tanto que una contracorriente superficial hacia el polo está presente a la altura dePunta Eugenia $\left(\sim 28^{\circ} \mathrm{N}\right)$. En esta área también se presenta una contracorriente profunda hacia el polo localizada sobre el talud continental en todas las estaciones del año, excepto en primavera (Hickey 1978, Lynn \& Simpson 1987, Durazo et al. 2010).

La presencia de remolinos anticiclónicos semipermanentes es evidente en los patrones de circulación superficial durante todo el año, excepto en otoño, de igual manera se observan estructuras de meandros al sureste de Isla Guadalupe (mejor definida en verano) y frente a Punta Eugenia (Soto-Mardones et al. 2004, Durazo et al. 2010). La importancia de estas estructuras de circulación, es que interrumpen la interconexión hidrodinámica entre las regiones norte y sur del área de estudio, interrupción que puede tener consecuencias notables en el transporte meridional de poblaciones biológicas (Durazo et al. 2010).

\section{ObTención de las MUeSTras}

Las larvas de $S$. japonicus fueron obtenidas a partir de 11 cruceros realizados a bordo del B/O 'Francisco de Ulloa', por el programa de Investigaciones Mexicanas de la Corriente de California (IMECOCAL): 3 en invierno (febrero 2006-2008); 4 en primavera (abril 2006-2008 y 2010); 2 en verano (agosto 2007 y julio 2008); y 2 en otoño (octubre 2008, septiembre 2009), con un total 722 muestras de zooplancton recolectadas. La distancia entre estaciones de muestreo fue de $\sim 37 \mathrm{~km}$ (20 $\mathrm{mn}$ ) y entre transectos perpendiculares a la línea de costa de $\sim 74 \mathrm{~km}$ (40 mn); el transecto más largo se extiende mar adentro hasta $200 \mathrm{~km}$ (108 mn; Fig. 1).

Las muestras de zooplancton fueron recolectadas con una red Bongo cilíndrico-cónica de $0,71 \mathrm{~m}$ de diámetro en la boca y malla de monofilamento de $505 \mu \mathrm{m}$, provistas de flujómetros (General Oceanics 2030RC), para medir el volumen de agua filtrada. Los arrastres fueron oblicuos desde la superficie hasta $200 \mathrm{~m}$ de profundidad máxima, a una velocidad de 2 nudos aproximadamente. Las muestras de plancton fueron preservadas en formaldehído al 4\%, neutralizado con una solución saturada de borato de sodio. De forma simultánea, en cada estación se realizaron mediciones continuas de conductividad y temperatura mediante un CTD (SBE-9/11), desde la superficie hasta el fondo o $1000 \mathrm{~m}$ de profundidad.
La medición de la composición de la biomasa del zooplancton se realizó mediante el método volumen desplazado del total del material recolectado en la red de $505 \mu \mathrm{m}$, después de remover los especímenes con volumen individual $>5 \mathrm{ml}$, y posteriormente se normalizó a $\mathrm{ml} 1000 \mathrm{~m}^{-3}$ (Smith \& Richardson 1977). El total de larvas de peces fue separado de las muestras de zooplancton, para ser preservadas en formaldehído al 1\%. La determinación taxonómica de las larvas de $S$. japonicus, se realizó de acuerdo a lo establecido por Kramer (1960). La abundancia de larvas se representó en número de larvas por $10 \mathrm{~m}^{2}$. Las larvas fueron medidas individualmente en su longitud estándar (LE) y altura del cuerpo (AC), con la ayuda de un microscopio estereoscopio Carl Zeiss, SV11, provisto de un micrómetro ocular.

\section{ANÁlisIS HIDROGRÁFICO}

Los datos de TSM $\left({ }^{\circ} \mathrm{C}\right)$ se obtuvieron de las mediciones realizadas con CTD (1 m de profundidad) interpolados a través del método de Kriging a una malla regular, con una resolución espacial de 6,66 x 6,66 km aproximadamente. Los mapas de circulación geostrófica se generaron a partir de los datos diarios 'delay time' (DT) de alturas dinámicas y sus componentes vectoriales, obtenidos del programa Archiving, Validation, and Interpretation of Satellite Oceanographic (AVISO) <http://www.aviso.altimetry.fr/>. Los mapas se presentaron con una resolución espacial de un tercio de grado, es decir aproximadamente $33 \times 33 \mathrm{~km}$. Los componentes vectoriales ' $u$ ' $y$ ' $v$ ' fueron utilizados para construir los flujos de corrientes expresados en velocidades de $\mathrm{cm} \mathrm{s}^{-1}$. Para corroborar que la información satelital fuera correcta, se hicieron mapas de geostrofía a partir de los datos del CTD con resultados similares. Debido a esto, y a la mayor cantidad de información, se prefirió utilizar la información satelital que permite una visión más amplia y una mejor representación de la circulación oceánica superficial de la zona. Los mapas resultantes son el promedio de los datos diarios para las fechas de cada crucero. En ellos, la distribución de la abundancia de larvas fue sobrepuesta, con la finalidad de llevar a cabo una exploración cualitativa de la influencia potencial del flujo de corrientes y otras estructuras (e.g., remolinos y meandros). Asimismo, la abundancia fue comparada con los mapas de la TSM in situ.

\section{Análisis de tallas y desarrollo}

Con el fin de identificar zonas propicias para el desarrollo larval de S. japonicus y realizar comparaciones, el área de estudio se dividió en 3 zonas: norte, centro y sur; basados en los resultados de la distribución y abundancia espacio-temporal de las larvas y la caracterización ambiental de la zona (Fig. 1). 
Para conocer si hubo diferencias significativas entre los promedios de las tallas con la estacionalidad, se realizó una prueba de Kruskal-Wallis y posteriormente un análisis de regresión lineal simple para observar la tendencia del desarrollo en cada zona-temporada. Donde el parámetro $B$ fue interpretado como el incremento en milímetros de la $\mathrm{AC}$ por cada milímetro de LE (AC/1 mm LE), y el error estándar de $\beta$ (EE) como la variación que puede presentarse en el incremento. Adicional a este análisis, se realizó una prueba ANCOVA para conocer si hubo diferencias significativas en los valores de incremento de AC de las larvas entre cada zona-temporada. Solo se realizó este análisis para cada zona-temporada que presentó un número de individuos recolectados, igual o mayor a 30 ejemplares. La proporción de la AC respecto de la LE, fue corroborada mediante un ajuste del Análisis de Medias Geométricas (AMG) propuesto por Ricker (1984), el cual se calculó con la siguiente ecuación:

$$
S \log Y=b S \log X
$$

donde $Y$ representó la medida de AC, $X$ la medida de LE en milímetros y $S$ la desviación estándar. La diferencia entre las desviaciones estándar de los logaritmos (representada por $b$ ), al diferir de 1 (es decir 1-b) pudo ser comparada con los valores $t$ de tablas para $N-2$ grados de libertad (con 95\% de confianza), con lo cual se determinó si existía o no un crecimiento alométrico en la relación AC/LE (Ricker 1984; Ortíz-Galindo et al. 2008). Cabe señalar que las larvas utilizadas en el análisis de regresión lineal simple y en el análisis de medias geométricas se presentaron en un intervalo de tallas de 2,2 a 13,2 mm LE. Las larvas de tallas mayores (>13,2 mm) fueron escasas y no formaron parte del análisis, porque estuvieron presentes en las zonas donde el número de individuos fue insuficiente para el análisis $(\mathrm{N}<30)$.

Con la premisa de que la temperatura y la disponibilidad del alimento son las variables con mayor influencia en el desarrollo larval de S. japonicus (Hunter \& Kimbrell 1980; Sassa \& Tsukamoto 2010, Weber \& McClatchie 2012), se realizó un análisis de cocientes para identificar los intervalos preferenciales en la distribución de las larvas. Las variables exploratorias (covariantes) fueron la TSM $\left({ }^{\circ} \mathrm{C}\right)$ in situ y los valores de la BZ $\left(1000 \mathrm{~m}^{-3}\right)$ de cada estación. El análisis de cocientes de la abundancia se calculó con la siguiente ecuación:

$$
Q_{i}=\frac{A_{i} / \sum_{i} A_{i}}{N_{i} / \sum_{i} N_{i}}
$$

donde $N_{i}$ y $A_{i}$ denotan, respectivamente, el número de estaciones y la abundancia total por clase $i$. Los valores de cociente mayores a $1\left(Q_{i}>1\right)$ indican la selección de hábitat positivo, es decir, intervalo de temperatura y volumen estimado de BZ óptimo (Ibaibarriaga et al. 2007).
Por último, para identificar las zonas y temporadas en que el ambiente favorece el desarrollo larval de S. japonicus, se comparó la tendencia del incremento de la AC/LE en el desarrollo larval, con los niveles de TSM y el volumen estimado de la BZ en que las larvas estuvieron presentes. Esta información se presentó con una sobreposición de los valores de incremento en gráficas de frecuencia de estas variables, para cada una de las zonas (norte, centro y sur), dentro de las temporadas analizadas (invierno, primavera y verano).

\section{Resultados}

\section{DiSTRIBUCIÓN ESPACIO-TEMPORAL DE LA ABUNDANCIA}

Durante las temporadas de invierno, las larvas de Scomber japonicus se presentaron solo en la zona sur y por lo general cerca de la costa, en un intervalo de TSM entre 18 y $20^{\circ} \mathrm{C}$, además de coincidir su distribución con los bordes de un aparente remolino anticiclónico, que es común en la época de invierno (Figs. 2 y 3 ).

En primavera, las larvas concurrieron en gran parte del área de estudio, tanto en estaciones oceánicas como costeras (excepto en la primavera de 2010), en un amplio intervalo térmico que incluye una menor TSM $\left(15-20^{\circ} \mathrm{C}\right)$. La mayor abundancia de larvas en esta temporada, se observó sobre la convergencia de formaciones de tipo remolinos frente a Punta Baja $\left(\sim 30^{\circ} \mathrm{N}\right)$ y en un remolino ciclónico relacionado con el efecto de punta que desvía el flujo hacia mar abierto frente a Punta Eugenia $\left(\sim 28^{\circ} \mathrm{N}\right)$, el cual fue más evidente en la primavera de 2008. De igual forma, se observó una zona de concentración de larvas en los bordes de una estructura anticiclónica en 2008 y 2010 en la zona sur (Figs. 2 y 3).

Durante los muestreos de verano, la mayor abundancia de larvas (10000 larvas $10 \mathrm{~m}^{-2}$ ) se presentó en la parte costera de la zona centro, en relación con un intervalo térmico más elevado $\left(19^{\circ}\right.$ y $\left.23^{\circ} \mathrm{C}\right)$. La distribución de las larvas pudo haber sido delimitada por 2 estructuras oceanográficas, una ciclónica y otra anticiclónica que tuvieron lugar frente a Punta Eugenia (verano de 2007) y un contraflujo serpenteante a lo largo del margen costero; que se aleja de la costa en la zona norte, el cual pareciera transportar agua hacia la costa frente a Punta Eugenia durante el verano de 2008 (Figs. 2 y 3).

Durante el otoño, no hubo registro de larvas de $S$. japonicus en toda el área de estudio; lo que fue coincidente con un incremento de la $\operatorname{TSM}\left(21^{\circ}\right.$ a $\left.27^{\circ} \mathrm{C}\right)$ y la intensificación de una contracorriente costera con dirección hacia el norte (Figs. 2 y 3 ). 


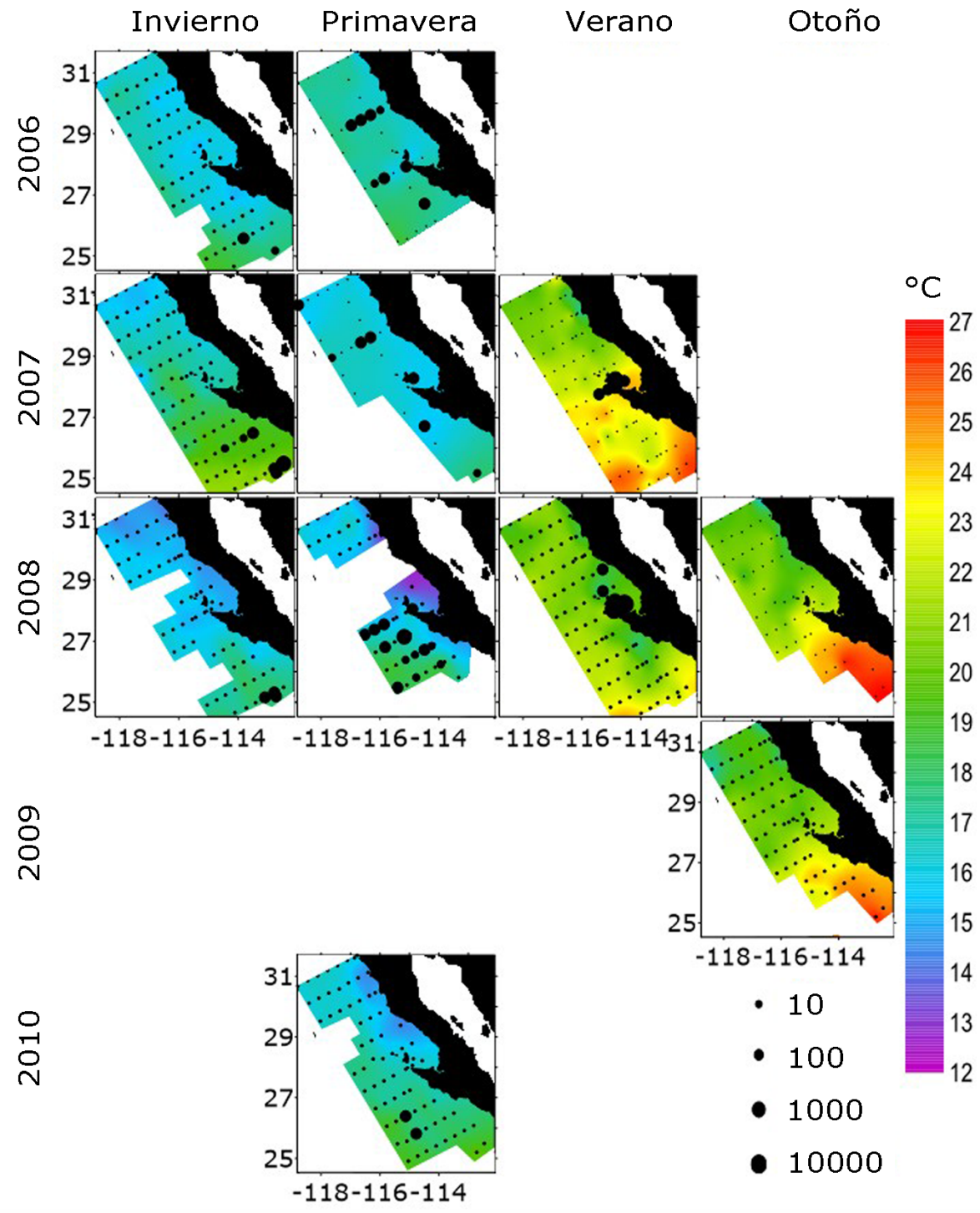

Figura 2. Distribución espacio-temporal de la temperatura y abundancia larval (larvas $10 \mathrm{~m}^{-2}$ ) de Scomber japonicus, para invierno, primavera, verano y otoño, durante el periodo de 2006 a 2010 / Spatio-temporal distribution of temperature and Scomber japonicus larval abundance (larvae $10 \mathrm{~m}^{-2}$ ), winter, spring, summer and autumn, during the period 2006 to 2010 


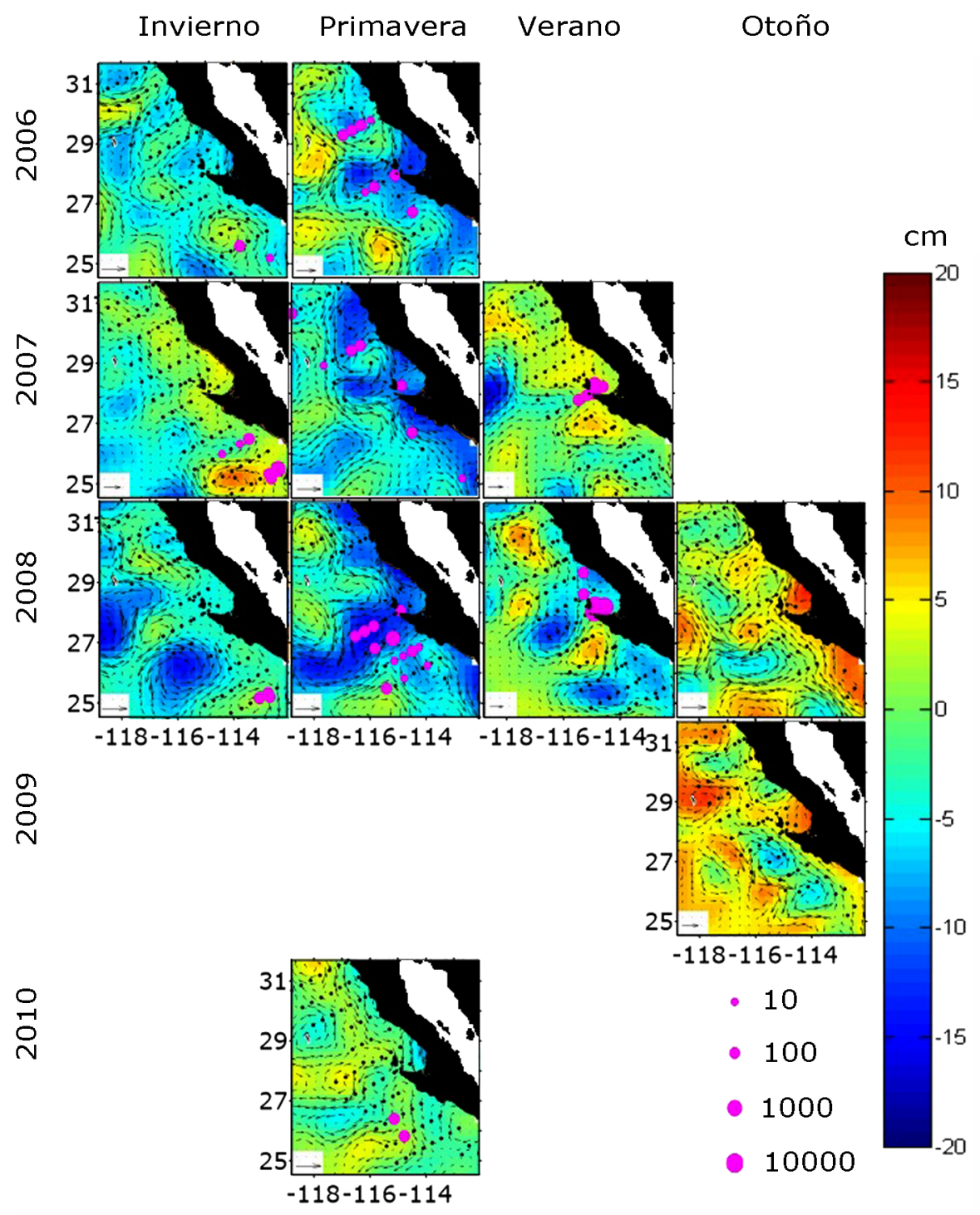

Figura 3. Distribución espacio-temporal de las anomalías en la altura del nivel medio del mar en centímetros (cm) y de la abundancia larval (larvas $10 \mathrm{~m}^{-2}$ ) de Scomber japonicus, para invierno, primavera, verano y otoño durante el periodo de 2006 a 2010. Las flechas identifican la dirección e intensidad del flujo de corrientes, referenciada a $25 \mathrm{~cm} \mathrm{~s}^{-1}$ en el recuadro inferior izquierdo de cada mapa / Spatiotemporal distribution of the mean sea level height anomalies in centimeters $(\mathrm{cm})$ and Scomber japonicus larval abundance (larvae $10 \mathrm{~m}^{-2}$ ), winter, spring, summer and autumn during the period 2006 to 2010. The arrows identify the current flow direction and intensity, referenced to $25 \mathrm{~cm} \mathrm{~s}^{-1}$ in the lower left panel of each map 


\section{Distribución TEMPoral de tallas larvales}

Durante los inviernos de 2007 y 2008 , el intervalo de tallas fue similar (3,4 a 8,6 mm; $\bar{X}=5,1 \mathrm{~mm}$ de LE; y 3,0 a 7,3 mm; $\overline{\mathrm{X}}=$ $4,5 \mathrm{~mm}$ de LE, respectivamente), y sin diferencias significativas $(P<0,05)$, pero el intervalo de tallas fue significativamente menor en invierno del 2006 ( 2,9 a 4,6 $\mathrm{mm} ; \overline{\mathrm{X}}=3,5 \mathrm{~mm}$ de $\mathrm{LE}$; Fig. 4a). Durante primavera de 2006 y 2007, el intervalo de tallas fue más amplio (2,6 a 12,1 y 2,7 a 20,3 mm de LE, respectivamente), pero más estrecho en primavera de 2010 (4,6 a 6,5 mm de LE). En esta temporada, no hubo diferencias significativas en los promedios de talla durante 2006, $2007 \mathrm{y}$ 2010 ( $\bar{X}=4,6,4,2$ y 5,5 mm de LE, respectivamente); con excepción de la primavera de 2008, que fue significativamente mayor (4,2 a 17,2 mm, $\bar{X}=9,0 \mathrm{~mm}$ LE; Fig. 4b). Por otra parte, en verano el intervalo de tallas fue similar en $2007 \mathrm{y}$ 2008 (2,6 a 9,3 y 2,6 a 8,4 mm LE, respectivamente) y sin diferencias significativas en los promedios de tallas $(\bar{X}=4,2$ y 4,3 mm LE, respectivamente; Fig. 4c).
Análisis de la relación altura del Cuerpo (AC) Y LONGITUD ESTÁNDAR (LE)

En la zona norte, durante la primavera de 2006 y 2007, las larvas de $S$. japonicus presentaron incrementos mínimos de la AC, por cada milímetro de LE (AC/1 mm LE), es decir, 0,273 y $0,256 \mathrm{~mm}$ de AC/1 mm LE, respectivamente; aunque con una amplia variabilidad representada por el error estándar del incremento (EE): 0,010 y 0,026 mm AC/1 mm LE, respectivamente. De forma similar, en la zona centro, en la primavera de 2008 se observó un bajo incremento $(0,257 \mathrm{~mm}$ AC/1 mm LE); mientras que en los veranos de 2007 y 2008, el incremento de la AC fue alto $(0,307$ y $0,292 \mathrm{~mm} \mathrm{AC/1} \mathrm{mm}$ LE, respectivamente). No obstante, en la zona centro, tanto en la primavera de 2008 como en los veranos de 2007 y 2008, siempre se observó poca variabilidad en el incremento de la $\mathrm{AC}(0,007,0,009$ y 0,003 mm AC/1 mm LE, respectivamente). En la zona sur, durante invierno de 2008, las larvas de la macarela presentaron el mayor incremento $(0,312 \mathrm{~mm}$ de $\mathrm{AC} /$ $1 \mathrm{~mm} \mathrm{LE})$, aunque con una amplia variabilidad $(0,015 \mathrm{~mm}$ de AC/1 mm LE). En cambio, en invierno del 2007 el incremento fue comparativamente menor que en 2008 (0,272 de AC/1 $\mathrm{mm} \mathrm{LE}$ ) y con poca variabilidad $(\mathrm{EE}=0,009 \mathrm{~mm}$ de $\mathrm{AC} / 1 \mathrm{~mm}$ LE) (Tabla 1). a) Invierno

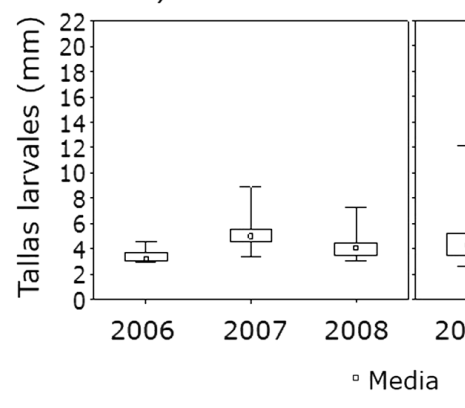

b) Primavera

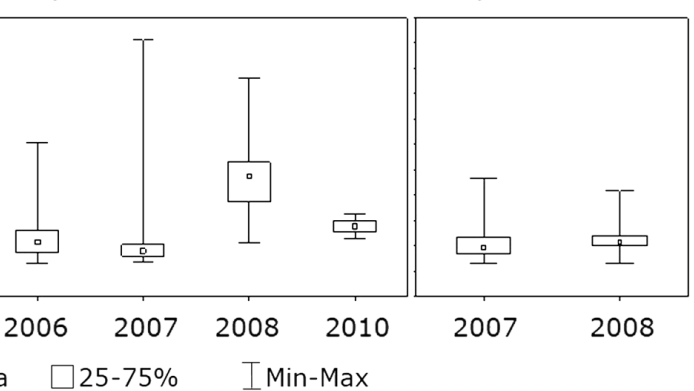

Figura 4. Diagramas de Caja-Bigotes de la distribución de tallas larvales de Scomber japonicus por temporada: a) Invierno; b) Primavera; y c) Verano del 2006 al 2010 / Box plots of larval sizes distribution of Scomber japonicus by season: a) Winter; b) Spring; and c) Summer during 2006 to 2010

Tabla 1. Parámetros del modelo de regresión simple para cada zona analizada: (ß) intercepto de la pendiente; (EEP) error estándar de la pendiente; (B) valor del incremento en milímetros por cada milímetro de LE; (EE) error estándar del incremento en milímetros; $\left(\mathbf{R}^{2}\right)$ valor de la variabilidad de los datos explicada por el modelo; valor de Fy estadístico P / Parameters of the simple regression model for each area analyzed: (B) intercept of the slope; (EEP) standard error of the slope; (B) value of the increase in millimeters per millimeter of SL; (EE) standard error of the increase in millimeters; $\left(R^{2}\right)$ value of data variability explained by the model; value of $F$ and $P$ statistical

\begin{tabular}{lccccccc}
\hline \multicolumn{1}{c}{ Modelo AC x LE } & $\alpha$ & EEP & $\beta$ & EE & $\mathrm{R}^{2}$ & $\mathrm{~F}$ & $P$ \\
\hline Norte-primavera 2006 & $-0,119$ & 0,050 & 0,273 & 0,010 &, 9408 & 684,77 & $<0,0001$ \\
Norte-primavera 2007 & 0,033 & 0,098 & 0,259 & 0,026 &, 7780 & 95,624 & $<0,0001$ \\
Centro-primavera 2008 & 0,105 & 0,066 & 0,257 & 0,007 &, 9362 & 1263,0 & $<0,0001$ \\
Centro-verano 2007 & $-0,138$ & 0,040 & 0,307 & 0,009 &, 9181 & 1111,7 & $<0,0001$ \\
Centro-verano 2008 & $-0,079$ & 0,014 & 0,292 & 0,003 &, 7603 & 7893,0 & $<0,0001$ \\
Sur-invierno 2007 & 0,029 & 0,049 & 0,272 & 0,009 &, 8074 & 810,20 & $<0,0001$ \\
Sur-invierno 2008 & $-0,167$ & 0,073 & 0,312 & 0,015 &, 9250 & 395,72 & $<0,0001$ \\
\hline
\end{tabular}


Tabla 2. Análisis de covarianza (ANCOVA) con valores de media ajustada y homogeneidad de pendiente (F) y valor $P$, que comprueban diferencias significativas de la relación morfométrica AC/ LE entre cada una de las zonas y temporadas / Analysis of covariance (ANCOVA) with values adjusted mean and slope homogeneity (F) and $\mathrm{P}$ value, which prove the significant differences in the AC/LE morphometric relationships between each of the areas and seasons

\begin{tabular}{ccccccc}
\hline & Media ajustada & & \multicolumn{2}{c}{$\begin{array}{c}\text { Homogeneidad } \\
\text { de pendiente }\end{array}$} \\
\cline { 1 - 2 } \cline { 5 - 6 } $\mathrm{F}$ & $P$ & & $\mathrm{~F}$ & $P$ \\
\hline 1,45 & 0,00000000000000509 & & 7,61 & 0,000000412 \\
\hline
\end{tabular}

\begin{tabular}{|c|c|c|c|}
\hline Modelo AC/ LE & Valor $1-b$ & Valor $t_{N-2}$ & $N$ \\
\hline Norte, primavera 2006 & 1,0258 & 1,6579 & 44 \\
\hline Norte, primavera 2007 & 1,0104 & 1,7011 & 30 \\
\hline Centro, primavera 2008 & 1,0017 & 1,6602 & 87 \\
\hline Centro, verano 2007 & 1,0155 & 1,6602 & 100 \\
\hline Centro, verano 2008 & 1,0171 & 1,645 & 2489 \\
\hline Sur, invierno 2007 & 1,0062 & 1,645 & 149 \\
\hline Sur, invierno 2008 & 1,0166 & 1,6839 & 33 \\
\hline
\end{tabular}

El modelo de regresión evidenció que el incremento de la $\mathrm{AC}$, con respecto a la LE en las larvas de $S$. japonicus, fue siempre lineal y altamente correlacionado (Fig. 5). Además, el ANCOVA mostró diferencias significativas en los incrementos, entre las zonas norte, centro y sur durante las épocas de invierno, primavera y verano de los años analizados $(P<0,05$ en la media ajustada y en la homogeneidad de la pendiente) (Tabla 2). Asimismo, en el análisis de medias geométricas se observó que ninguno de los valores de $1-b$ fue mayor que el valor teórico con grados de libertad $N$-2; esto demuestra que el desarrollo de las larvas de macarela en la relación AC/LE, siempre mostró un crecimiento isométrico (Tabla 3).

Cabe señalar que las larvas utilizadas en el análisis de regresión lineal simple y en el análisis de medias geométricas se presentaron en un intervalo de tallas de 2,2 a 13,2 mm LE. Las larvas de tallas mayores (>13,2 $\mathrm{mm})$ fueron escasas y no formaron parte del análisis, porque estuvieron presentes en las zonas donde el número de individuos fue insuficiente $(\mathrm{N}<30)$.

Relación del DESARROLlo de S. JAPONICUS CON LA TEMPERATURA DEL AMBIENTE Y BIOMASA DEL ZOOPLANCTON

Las larvas de S. japonicus, de acuerdo con lo indicado por el análisis de cocientes, se presentaron en un intervalo preferencial de 15 a $22^{\circ} \mathrm{C}$ de $\mathrm{TSM}$ (excepto a $17^{\circ} \mathrm{C}$ ), mientras que las larvas concurren en diferentes niveles preferenciales de la BZ (1, 300, 700 y $1000 \mathrm{ml} 1000 \mathrm{~m}^{-3}$; Fig. 6).

El incremento en la AC de las larvas con respecto a la TSM, fue comparativamente menor durante la primavera $(0,257$ $0,273 \mathrm{~mm}$ ), en temperaturas entre 15 y $20^{\circ} \mathrm{C}$. Al contrario del verano, cuando se observó un mayor incremento (0,292-0,307 $\mathrm{mm}$ ), que coincidió con un ligero aumento en la temperatura $\left(16-22^{\circ} \mathrm{C}\right)$. En las épocas de invierno, aunque los incrementos fueron distintos para las larvas del 2007 y 2008 (0,272 y 0,312 $\mathrm{mm}$, respectivamente), estas se presentaron en temperaturas de entre $18-20$ y $17^{\circ} \mathrm{C}$, respectivamente (Fig. 7).

Por otro lado, la variación en los niveles de BZ y la latitud geográfica se relacionaron con los cambios en la variabilidad del incremento de la AC. En la zona norte y sur, cuando las larvas presentan una mayor variabilidad en el incremento de talla $(0,009-0,026 \mathrm{~mm})$, los niveles de BZ fueron menores a $400 \mathrm{ml} 1000 \mathrm{~m}^{-3}$; mientras que en la zona centro, se registró una menor variabilidad en el incremento de tallas $(0,003-0,009$ $\mathrm{mm})$, los niveles de BZ fueron más altos (> $500 \mathrm{ml} 1000 \mathrm{~m}^{-3}$; Fig. 8).

\section{Discusión}

Las larvas de Scomber japonicus presentaron una marcada diferencia estacional en su distribución y abundancia. Durante las temporadas de invierno, se presentaron solo en la zona sur (frente al Golfo de Ulloa, $\sim 26^{\circ} \mathrm{N}$ ), mientras que en las temporadas de primavera la distribución se extendió por toda el área de estudio, incluso en las estaciones más oceánicas, pero sus abundancias fueron comparativamente menores durante invierno y primavera (10 y 100 larvas $10 \mathrm{~m}^{-2}$ ). En contraste, durante las temporadas de verano las larvas alcanzaron la más alta abundancia (1000 larvas $10 \mathrm{~m}^{-2}$ ); no obstante, su distribución fue principalmente costera frente a Punta Eugenia $\left(\sim 28^{\circ} \mathrm{N}\right)$. Al respecto, Moser et al. (1993) señalan que es común observar dos incrementos de la abundancia larval frente a la COPBC, uno en invierno frente a Bahía Magdalena y otro más abundante en verano alrededor de Punta Eugenia $\left(28^{\circ} \mathrm{N}\right)$. 


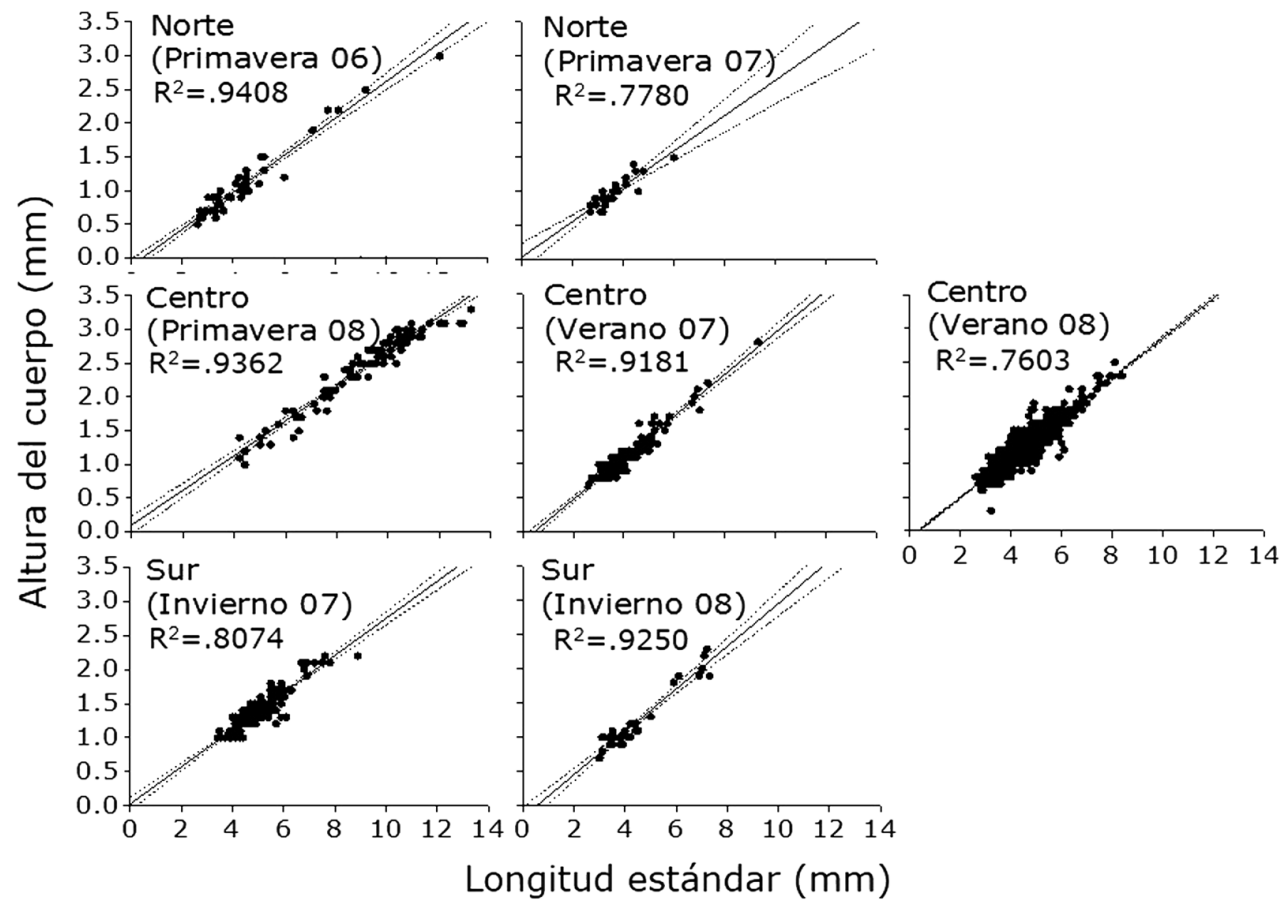

Figura 5. Relación lineal de la altura del cuerpo en relación a la longitud estándar de Scomber japonicus, para la zona norte, centro y sur; durante invierno, primavera y verano de los años analizados (ver desglose de las ecuaciones en Tabla 1) / Lineal relation of increase in body height with respect to standard length of Scomber japonicus, in the north, central and south zones, during winter, spring and summer of the years analyzed (See details of the equations in Table 1)
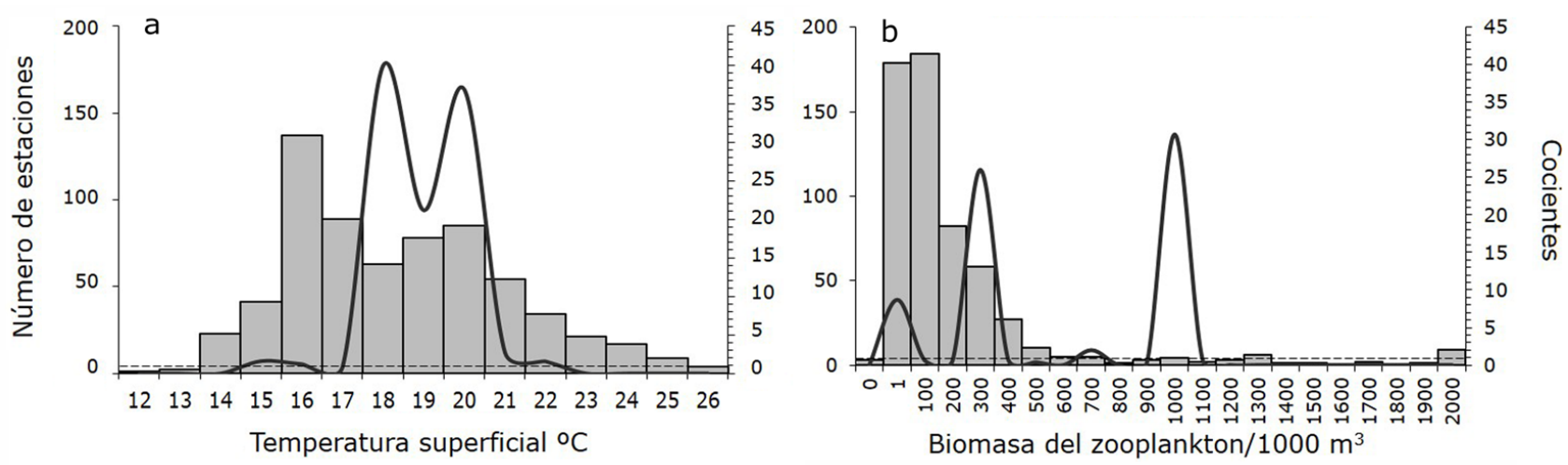

Figura 6. Análisis de cocientes de la abundancia larval de Scomber japonicus: a) con respecto a la temperatura superficial del mar y b) la biomasa del zooplancton. Las barras verticales indican el número total de estaciones muestreadas por intervalo de clase de temperatura y biomasa del zooplancton. Los valores de cociente $>1$ indican preferencia (línea continua, por encima de la línea punteada) / Quotient analysis of Scomber japonicus larval abundance with respect to: a) sea surface temperature; and b) zooplankton biomass. Vertical bars indicate the total sampling effort by class interval of temperature and zooplankton biomass. Quotient values $>1$ indicate larval preference (solid line, for above dashed line) 


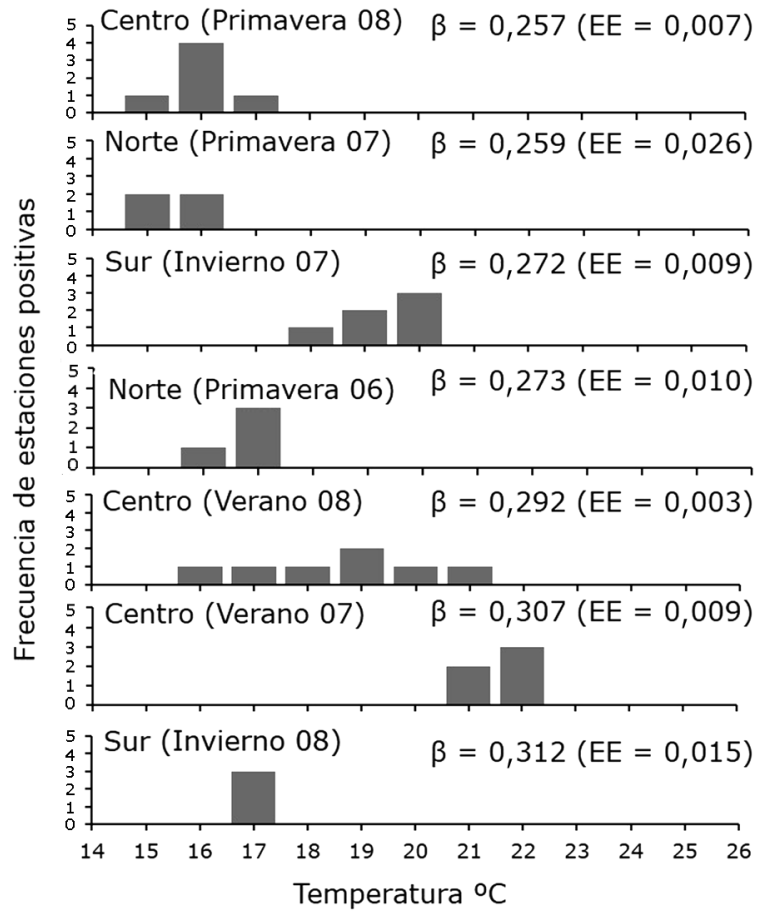

Figura 7. Frecuencia de estaciones positivas (barras) de larvas de Scomber japonicus por intervalo de temperatura $\left({ }^{\circ} \mathrm{C}\right)$, y el incremento de la altura del cuerpo con respecto a la longitud estándar en milímetros ( $\beta$ ) por zona y temporada. EE indica la variación del incremento / Positive stations (frequency, bars), of Scomber japonicus larvae by temperatura interval $\left({ }^{\circ} \mathrm{C}\right)$, and body height increase with respect to the standard length in millimeters $(\beta)$, by zone and season. $\mathrm{EE}$ indicates the variation of the increment

La diferencia en tiempo y espacio en la distribución de las larvas de $S$. japonicus, sugiere la presencia de diferentes stocks. Esto es consistente con la identificación previa de 2 stocks frente a la COPBC (Gluyas-Millán \& QuiñónezVelázquez 1996): uno al sur del Golfo de Ulloa $\left(\sim 24,5^{\circ} \mathrm{N}\right)$ que es más evidente durante el invierno (Arcos-Huitrón \& TorresVillegas 1990), y otro frente a Bahía Sebastián Vizcaíno $\left(\sim 28^{\circ} \mathrm{N}\right)$, cuya distribución puede ser amplia durante primavera y posteriormente, el stock se concentra en Bahía Sebastián Vizcaíno en verano (Gluyas-Millán 1994). En apoyo a la existencia de los 2 stocks de la macarela del Pacífico, la amplia distribución de larvas observada en primavera sugiere que pudiera corresponder a un stock norteño, relacionado con temperaturas comparativamente menores $\left(15-20^{\circ} \mathrm{C}\right)$, y posteriormente durante el verano con temperaturas entre $19 \mathrm{y}$ $23^{\circ} \mathrm{C}$, la distribución de los productos del desove se restringe a la zona costera frente Bahía Sebastián Vizcaíno $\left(\sim 28^{\circ} \mathrm{N}\right)$. En cambio, la presencia de larvas restringidas a la porción sur del área de estudio durante el invierno (Golfo de Ulloa, $\sim 26^{\circ} \mathrm{N}$ ),

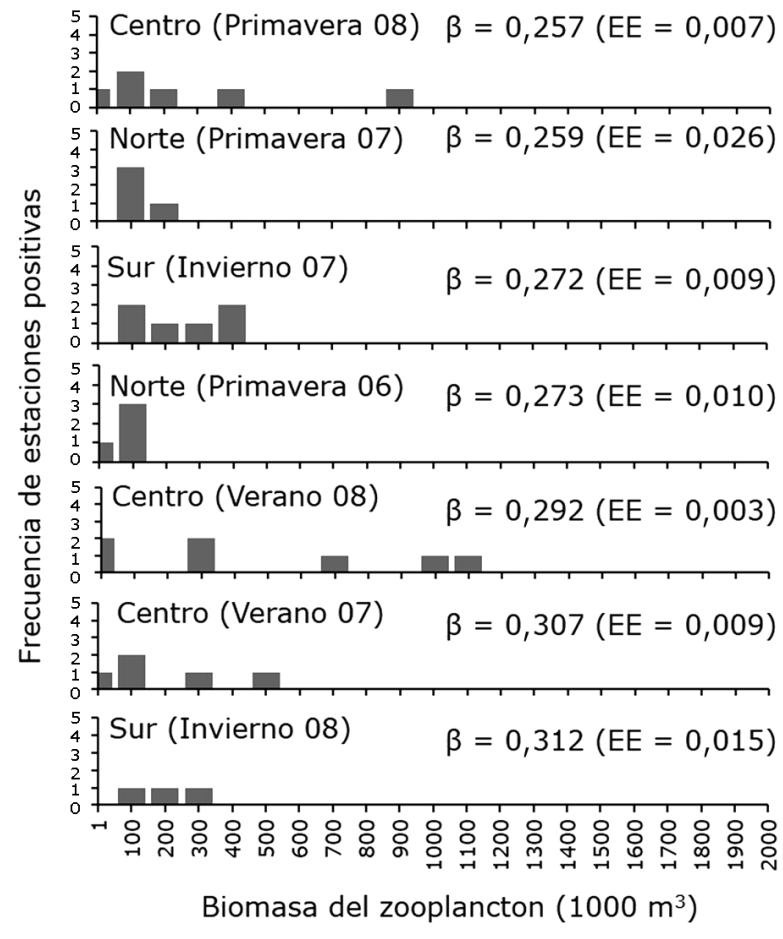

Figura 8. Frecuencia de estaciones positivas (barras) de larvas de Scomber japonicus por intervalo de biomasa del zooplancton (1000 $\mathrm{m}^{-3}$ ), y el incremento de la altura del cuerpo con respecto a la longitud estándar en milímetros $(\beta)$ por zona y temporada. EE indica la variación del incremento / Positive stations (frequency, bars), of Scomber japonicus larvae by zooplankton biomass $\left(1000 \mathrm{~m}^{-3}\right)$, and body height increase with respect to the standard length in millimeters $(\beta)$, by zone and season. EE indicates the variation of the increment

pudiera corresponder a un stock sureño relacionado con temperaturas de $18-20^{\circ} \mathrm{C}$. Este intervalo coincide con el determinado en Bahía Magdalena $\left(18,5-23,5^{\circ} \mathrm{C}\right.$, FunesRodríguez et al. 2007) y en el Golfo de California $\left(16-22^{\circ} \mathrm{C}\right.$; Esqueda-Escárcega 1995) y por consiguiente, los organismos que se distribuyen al sur de la COPBC, podrían corresponder a este stock sureño tal como lo han señalado previamente Gluyas-Millán \& Quiñónez-Velázquez (1996) y FunesRodríguez et al. (2007).

El intervalo preferencial de temperatura donde se presentaron las larvas de macarela del Pacífico, fue similar al reportado para todo el sistema de la CC $\left(14\right.$ a $\left.21,9^{\circ} \mathrm{C}\right)$ (Kramer 1960, Weber \& McClatchie 2012), incluso en la distribución de larvas de la macarela del sur del Mar de China (Sassa \& Tsukamoto 2010). No obstante, la copiosa agregación de larvas en la zona costera frente a Punta Eugenia $\left(28^{\circ} \mathrm{N}\right)$ en verano, semeja la existencia de un hábitat apropiado, con temperaturas entre 19 y $23^{\circ} \mathrm{C}$. Hunter \& Kimbrell (1980) señalaron que las 
larvas de macarela tienden a completar exitosamente su desarrollo en este intervalo. Por el contrario, se asume que evitan temperaturas mayores de $23^{\circ} \mathrm{C}$, a juzgar por la ausencia de larvas y las temperaturas elevadas durante el otoño; además de una disminución de la actividad reproductiva de la especie que tiene lugar durante esta temporada (Kramer 1969).

El efecto de los remolinos ciclónicos de mesoescala ha sido señalado como precursor en la retención y probable reducción de la mortalidad en larvas de diferentes especies pelágicocosteras, como la sardina Monterrey y anchoveta norteña, al sur de California (Logerwell \& Smith 2001, Asch \& Checkley 2013); al igual que los flujos de las corrientes, remolinos y meandros, pudieran ser responsables del transporte y retención en organismos de plancton en la COPBC (Funes-Rodríguez et al. 2015), como en el caso de la macarela y otros escómbridos en otras regiones del océano mundial (Alemany et al. 2010, Sassa \& Tsukamoto 2010). Durante la primavera, el transporte de larvas hacia el sur con el flujo de la CC y probablemente, el transporte oceánico por el efecto de punta y el transporte de Ekman (Parrish et al. 1981, Norcross \& Shaw 1984), pudieran ser la causa de una amplia distribución latitudinal de las larvas y su distanciamiento de la costa frente a Punta Eugenia $\left(\sim 28^{\circ} \mathrm{N}\right)$. De forma simultánea, una zona de retención está presente frente a Punta Baja en primavera $\left(\sim 30^{\circ} \mathrm{N}\right)$, al parecer relacionada con una convergencia entre un aparente remolino ciclónico y otro anticiclónico.

De manera similar, durante el verano una importante zona de retención costera se presenta en los alrededores de Bahía Sebastián Vizcaíno $\left(\sim 28^{\circ} \mathrm{N}\right)$, aparentemente asociada a un remolino ciclónico y otro anticiclónico hacia el sur $\left(\sim 27^{\circ} \mathrm{N}\right)$; que además parece evitar la dispersión de sus larvas hacia mar abierto, debido al contraflujo serpenteante de la contracorriente costera hacia el polo (Parrish et al. 1981, Norcross \& Shaw 1984). Esto es común de observar como resultado del debilitamiento en el transporte del agua de la CC, relacionado con los cambios en la circulación oceánica en el extremo sur de la CC (Durazo et al. 2010).

Durante las temporadas de invierno y verano, las larvas fueron de menor tamaño y no superaron los $9 \mathrm{~mm}$ de LE; mientras que en las temporadas de primavera (2006, $2007 \mathrm{y}$ 2008), alcanzaron tallas de hasta 20,3 mm de LE. De acuerdo con Folkvord et al. (2009) la diferencia en la variabilidad de tallas está influenciada por la temperatura y disponibilidad de las presas (i.e., entre mayor sea la variación de estos factores, mayor será la variación de las tallas, siempre y cuando no se excedan los límites de tolerancia). Esto pudiera estar relacionado con la amplia variabilidad de tallas observada en las temporadas de primavera y verano, asociadas a un amplio intervalo de TSM en primavera $\left(15-20^{\circ} \mathrm{C}\right)$, aunque ligeramente más cálido en verano $\left(16-23^{\circ} \mathrm{C}\right)$; por el contrario, las tallas pequeñas se presentaron en las temporadas de invierno en un intervalo estrecho de temperatura $\left(18-20^{\circ} \mathrm{C}\right)$. Por su parte, Matsuura $\&$ Hewitt (1995) mencionaron que la agregación de larvas de S. japonicus con tallas similares dentro de un hábitat, reduce el canibalismo y por lo tanto aumenta las probabilidades de supervivencia.

Cabe señalar que la información de los muestreos analizados (2006-2009), se ubica en un periodo caracterizado por anomalías negativas en el índice multivariado El Niño (Climate Diagnostic Bulletin, NOAA <http://www.esrl.noaa.gov/psd/ enso/mei/index.html >), con excepción de la primavera del 2010, con un evento El Niño moderado que influyó en una disminución de la abundancia larval y también una menor variabilidad de tallas estadísticamente significativa.

Por otro lado, el rápido crecimiento larval de $S$. japonicus es una característica de las larvas de los escómbridos, por lo que requieren de una temperatura óptima y una adecuada alimentación (Hunter \& Kimbrell 1980). La temperatura no sólo determina la velocidad del crecimiento, también cambia el patrón de desarrollo, a menor temperatura el desarrollo se prolonga y viceversa, a temperaturas más altas se genera un inicio temprano de la alimentación exógena y la natación (Pörtner et al. 2010). Un hábitat apropiado para el desarrollo larval, proporciona una temperatura óptima además de altos niveles de presas, lo cual permite un rápido desarrollo y una mayor probabilidad de supervivencia (Hunter \& Kimbrell 1980, Werner 2002, Kanaji et al. 2009).

La relación morfométrica AC/LE es considerada como un buen indicador de la condición nutricional y del desarrollo de las larvas de peces (Ehrlich et al. 1976, Theilacker 1978, Yúfera et al. 1993). A diferencia de las técnicas histológicas y bioquímicas, las técnicas morfométricas, no requieren de una preparación especial de los especímenes (Theilacker 1978, Catalán-Alemani 2003). La alta disponibilidad de alimento interpretada a partir de la BZ y el incremento de temperatura frente a Punta Eugenia $\left(\sim 28^{\circ} \mathrm{N}\right)$ durante la época de verano, pudieran ser las causas de un mayor incremento en la AC, como es indicado por una baja variabilidad en el error estándar $(\mathrm{EE}=0,003-0,009 \mathrm{~mm})$ y esto mismo pudiera reflejar una estabilidad en el hábitat y un mejor desarrollo de los organismos. A pesar de que la BZ no refleja el tamaño, ni la calidad del alimento, su empleo está altamente correlacionado con la biomasa del microplancton como un índice confiable de la disponibilidad del alimento (Sinclair et al. 1985). Lo anterior resulta importante si se considera que las larvas de macarela se alimentan principalmente de nauplios de copépodos, cladóceros, apendicularias y eventualmente de diatomeas (Sánchez-Velasco \& Shirasago 2000), mientras que para otras 
especies de perciformes son principalmente los nauplios de copépodos y cladoceros (Turner 1984). En el área de estudio, los copépodos son dominantes $(>50 \%)$ y presentan una distribución principalmente costera, mientras que las apendicularias, aunque en menor abundancia, son comunes en los alrededores de Punta Eugenia (Lavaniegos et al. 2009a, b, 2010).

En los periodos de primavera, aun cuando las larvas presentaron tallas mayores, el valor del incremento de la AC fue menor y muy variable. Una menor tasa de incremento en la AC durante el desarrollo larval en otras especies de peces (e.g., Trachurus symmetricus y Sparus aurata), es el reflejo de la ausencia de alimento y un probable evento de inanición (Theilacker 1978, Yúfera et al. 1993). Los bajos incrementos de la AC con una mayor variabilidad (EE) pudieran indicar condiciones no optimas de alimentación (Ehrlich et al. 1976, Hunter \& Kimbrell 1980, Pörtner et al. 2010) lo que provoca un lento crecimiento (como se pudo observar en la zona sur, invierno; norte y centro, primavera). Al respecto, la distribución de los copépodos fue amplia a lo largo del área de estudio en invierno y primavera (Lavaniegos et al. 2009a, b, 2010). Sin embargo, la distribución de larvas de macarela no coincidió con los centros de mayor abundancia de copépodos.

Cabe señalar que el crecimiento estimado para S. japonicus fue de tipo isométrico, en la relación AC/LE obtenida con el AMG (Ricker 1984) aun cuando existió una diferencia en los valores del incremento de la AC para cada zona y temporada. El crecimiento isométrico en larvas fusiformes, es una estrategia que caracteriza a las larvas depredadoras y de rápido crecimiento como en $S$. japonicus y Eugerres lineatus (Hunter 1981, Ortíz-Galindo et al. 2008). Sin embargo, si el alimento es escaso el crecimiento isométrico se interrumpe y las larvas presentan evidencias de inanición, hasta alcanzar el 'punto de no retorno’ debido al alto consumo de energía (Froese 1990).

Los resultados de este estudio permitieron determinar que durante primavera, a pesar de estar ampliamente distribuidas las larvas del stock norteño, presentan un menor desarrollo en la proporción morfométrica altura del cuerpo/longitud estándar; en relación con un reducido intervalo de temperaturas (15$17^{\circ} \mathrm{C}$ ) y niveles de BZ comparativamente bajos ( $<400 \mathrm{ml} 1000$ $\mathrm{m}^{-3}$ ). Durante el verano, con la contracción de este stock en el área de Punta Eugenia $\left(\sim 28^{\circ} \mathrm{N}\right)$, las larvas presentan un mayor incremento en la altura del cuerpo, al encontrarse en un amplio intervalo de temperaturas $\left(16-22^{\circ} \mathrm{C}\right)$ con niveles de biomasa del zooplancton que pueden llegar a más de $500 \mathrm{ml} 1000 \mathrm{~m}^{-3}$.

\section{Agradecimientos}

Los autores expresan su agradecimiento al equipo del laboratorio de Plancton del Centro de Investigación Científica y Educación
Superior de Ensenada, por la recolección y procesamiento de muestras. Al personal del Departamento de Plancton y Ecología Marina del Centro Interdisciplinario de Ciencias Marina (CICIMAR-IPN). EAG recibió beca CONACYT y BEIFI. AFGA agradece los apoyos de los programas EDI y COFAAIPN y SNI CONACYT. Este trabajo fue apoyado por el programa IMECOCAL(CONACYT: G0041T; 017Pñ-1297 y G35326T) e IPN (SIP: 20150637 y 20160225). Los productos de altimetría fueron obtenidos por medio de Archiving, Validation, and Interpretation of Satellite Oceanographic (AVISO) <http://www.aviso.altimetry.fr/> .

\section{LITERATURA CITADA}

Alemany F, L Quintanilla, P Velez-Belchí, A García, D Cortés, JM Rodríguez, ML Fernández de Puelles, C González-Pola \& JL López-Jurado. 2010. Characterization of the spawning habitat of Atlantic bluefin tuna and related species in the Balearic Sea (Western Mediterranean). Progress in Oceanography 86(1-2): 21-38.

Arcos-Huitrón NE \& JR Torres-Villegas. 1990. Ciclo reproductor de la macarela del Pacífico Scomber japonicus Houttuyn (Pisces: Scombridae) en Bahía Magdalena, Baja California Sur, México. Investigaciones Marinas CICIMAR 5(1):37-45.

Asch RG \& DM Checkley. 2013. Dynamic height: a key variable for identifying the spawning habitat of small pelagic fishes. Deep-Sea Research Part I 71: 79-91.

Buckley LJ. 1984. RNA-DNA ratio: an index of larval fish growth in the sea. Marine Biology 80: 291-298.

Catalán-Alemani IA. 2003. Condition indices and their relationship with environmental factors in fish larvae. Tesis Doctoral, Facultad de Biología, Universidad de Barcelona, Barcelona, $265 \mathrm{pp}$.

Chicharo MA. 1998. Nutritional condition and starvation in Sardina pilchardus (L.) larvae off southern Portugal compared with some environmental factors. Journal of Experimental Marine Biology and Ecology 225: 123-137.

Clemmesen CM \& T Doan. 1996. Does otolith structure reflect the nutritional condition of a fish larva? Comparison of otolith structure and biochemical index (RNA/DNA ratio) determined on cod larvae. Marine Ecology Progress Series 138:33-39.

Durazo R, AM Ramírez, LE Miranda \& LA SotoMardones. 2010. Climatología de variables hidrográficas. En: Gaxiola-Castro G \& R Durazo (eds). Dinámica del ecosistema pelágico frente a Baja California, 1977-2007: diez años de Investigaciones Mexicanas de la Corriente de California, pp. 25-57. Secretaría de Medio Ambiente y Recursos Naturales, México D. F.

Ehrlich KF, JHS Blaxter \& R Pemberton. 1976. Morphological and histological changes during the growth and starvation of herring and plaice larvae. Marine Biology 35: $105-118$. 
Esqueda-Escarcega GM. 1995. Spatial and temporal distribution of Scomber japonicus larvae in the Sea of Cortez (1984-1988). Scientia Marina 59(3-4): 391-397.

Ferron A \& WC Leggett. 1994. An appraisal of condition measures for marine fish larvae. Advances in Marine Biology 30:217-303.

Folkvord A, Ø Fiksen, H Høie, A Johannessen, E Otterlei \& KW Vollset. 2009. What can size distributions within cohorts tell us about ecological processes in fish larvae? Scientia Marina 73: 119-130.

Froese R. 1990. Growth strategies of fish larvae. ICES C.M./ L: 91: 1-20.

Funes-Rodríguez R, JD Leal-Espinoza, A HinojosaMedina, ME Hernández-Rivas \& C Flores-Coto. 2007. Composición, distribución y abundancia de larvas de peces en Bahía Magdalena. En: Funes-Rodríguez R, J GómezGutiérrez \& JR Palomares-García (eds). Estudios ecológicos en Bahía Magdalena, pp. 205-219. CICIMAR-IPN, La Paz.

Funes-Rodríguez R, JA Ruíz-Chavarría, R GonzálezArmas, R Durazo \& SA Guzmán-del Proó. 2015. Influence of hydrographic conditions on the distribution of Spiny lobster (Panulirus interruptus) larvae off the west coast of the Baja California Peninsula. Transactions of the American Fisheries Society 144(6): 1192-1205.

Gluyas-Millán MG. 1994. Reproducción, edad y talla de primera madurez de la macarela Scomber japonicus (Houttuyn, 1872) en Bahía Vizcaíno. Scientia Marina 20(3): 409-419.

Gluyas-Millán MG \& C Quiñónez-Velázquez. 1996. Evidencias de distintos grupos poblacionales de macarela Scomber japonicus. Scientia Marina 22(3): 377-395.

Hare JA \& RK Cowen. 1997. Size, growth, development, and survival of the planktonic larvae of Pomatomus saltatrix (Pisces: Pomatomidae). Ecology 78(8): 2415-2431.

Hickey BM. 1979. The California Current System-hypotheses and facts. Progress in Oceanography 8(4): 191-279.

Hsieh Ch-H, C Reiss, W Watson, MJ Allen, JR Hunter, RN Lea, RH Rosenblatt, PE Smith \& G Sugihara. 2005. A comparison of long-term trends and variability in population of larvae of exploited and unexploited fishes in the Southern California regions: A community approach. Progress in Oceanography 67: 160-185.

Hunter JR. 1981. Feeding ecology and predation of marine fish larvae. In: Lasker R (ed). Marine fish larvae: morphology, ecology, and relation to fisheries, pp. 33-77. Washington Sea Grant Program, Seattle.

Hunter JR \& CM Kimbrell. 1980. Early life history of Pacific mackerel. Scomber japonicus. Fishery Bulletin 78(1): 89101.

Ibaibarriaga L, X Irigoien, M Santos, I Motos, JM Fives, C Franco, A Lago de Lanzós, S Acevedo, M Bernal, N Bez, G Eltink, A Farinha, C Hammer, SA Iversen, SP Milligan \& DG Reid. 2007. Egg and larval distributions of seven fish species in north-east Atlantic waters. Fisheries Oceanography 16(3): 284-293.
Iles TD \& M Sinclair. 1982. Atlantic herring: Stock discreteness and abundance. Science 215: 627-633.

Kanaji Y, Y Watanabe, T Kawamura, S Xie, Y Yamashita, C Sassa \& Y Tsukamoto. 2009. Multiple cohorts of juvenile jack mackerel Trachurus japonicus in waters along the Tsushima Warm Current. Fisheries Research 95: 139-145.

Kramer D. 1960. Development of eggs and larvae of Pacific mackerel and distribution and abundance of larvae 1952-1956. Fishery Bulletin 60: 393-438.

Kramer D. 1969. Synopsis of the biological data on the Pacific mackerel, Scomber japonicus, Houttuyn (Northeast Pacific). FAO Fisheries Synopsis 40: 1-20.

Lavaniegos BE, JL Cadena-Ramírez \& P García-García. 2009a. Zooplankton biomass and structure off the western coast of Baja California during 2006 (IMECOCAL cruises 0602, 0604, 0607). Informe Técnico. Departamento de Oceanografía Biológica. CICESE 96023: 50.

Lavaniegos BE, JL Cadena-Ramírez \& P García-García. 2009b. Zooplankton biomass and structure off the western coast of Baja California during 2007 (IMECOCAL cruises 0701, 0704, 0708). Informe Técnico. Departamento de Oceanografía Biológica, CICESE 96950: 51.

Lavaniegos BE, JL Cadena-Ramírez, O Molina-González \& P García-García. 2010. Zooplankton biomass and structure off the western coast of Baja California during 2008 (IMECOCAL cruises 0801, 0804, 0807, 0810). Informe Técnico, Departamento de Oceanografía Biológica, CICESE 999407: 1-54.<http://imecocal.cicese.mx/publicaciones/ informes/zooplancton/zoo_2008.pdf >

Li S \& J Mathias. 1987. The critical period of high mortality of larval fish - a discussion based on current research. Chinese Journal of Oceanology and Limnology 5(1): 81-96.

Lo NCH, E Dorval, R Funes-Rodríguez, ME HernándezRivas, Y Huang \& Z Fan. 2010. Utilities of larval densities of Pacific mackerel (Scomber japonicus) off California, U.S.A. and west coast of Mexico from 1951 to 2008, as spawning biomass indices. Ciencia Pesquera 18(2): 59-75.

Logerwell EA \& PE Smith. 2001. Mesoscale eddies and survival of late stage Pacific sardine (Sardinops sagax) larvae. Fisheries Oceanography 10(1): 13-25.

Lynn RJ \& JJ Simpson. 1987. The California Current Systemthe seasonal variability of its physical characteristics. Journal of Geophysical Research 92(C12): 12.947-12.966.

Matsuura Y \& R Hewitt. 1995. Changes in the spatial patchiness of Pacific mackerel, Scomber japonicus, larvae with increasing age and size. Fishery Bulletin 93(1): 172178.

Moser HG, RL Charter, PE Smith, DA Ambrose, SR Charter, CA Meyer, EM Sandknop \& W Watson. 1993. Distributional atlas of fish larvae and eggs in the California Current Region: Taxa with 1000 or more total larvae, 1951 through 1984. California Cooperative Fisheries Investigations, Atlas 31: 1-233. 
Norcross BL \& RF Shaw. 1984. Oceanic and estuarine transport of fish eggs and larvae: A review. Transactions of the American Fisheries Society 113: 153-165.

O'Connell CP. 1976. Histological criteria for diagnosing the starving condition in early post yolk sac larvae of the northern anchovy, Engraulis mordax Girard. Journal of Experimental Marine Biology and Ecology 25: 285-312.

Ortíz-Galindo JL, JL Castro-Aguirre, EF Balart \& NA Pliego. 2008. Description of early ontogeny and osteological development of streaked mojarra Eugerres lineatus (Teleostei: Percoidei:Gerreidae). Zootaxa 1911: 1-30.

Parrish RH, CS Nelson \& A Bakun. 1981. Transport mechanisms and reproductive success of fishes in the California Current. Biological Oceanography 1(2): 175-203.

Pörtner HO, PM Schulte, CM Wood \& F Schiemer. 2010. Niche dimensions and limits in fishes: an integrative view. Illustrating the role of physiology in understanding ecological realities. Physiological and Biochemical Zoology 83: 808-826.

Ricker WE. 1984. Computation and uses of central trend lines. Canadian Journal of Zoology 62: 1897-1905.

Sánchez-Velasco L \& B Shirasago. 2000. Larval feeding of Scomber japonicus (pisces: Scombridae) in the Gulf of California and its relation to temperature and chlorophyll. Pacific Science 54(2): 127-136.

Sassa C \& Y Tsukamoto. 2010. Distribution and growth of Scomber japonicus and $S$. australasicus larvae in the southern East China Sea in response to oceanographic conditions. Marine Ecology Progress Series 419(30): 185199.

Sinclair M. 1988. Marine populations. An essay on population regulation and speciation, pp. 252. University of Washington Press. Seattle.

Sinclair M, MJ Tremblay \& P Bernal. 1985. El Niño events and variability in a Pacific mackerel (Scomber japonicus) survival index: support for Hjort's second hypothesis. Canadian Journal of Fisheries and Aquatic Sciences 42: 602608 .
Smith PE \& SL Richardson. 1977. Standard techniques for pelagic fish egg and larva surveys. FAO Fisheries Technical Paper 175: 1-100.

Soto-Mardones L, A Parés-Sierra, J García, R Durazo \& S Hormazabal. 2004. Analysis of the mesoscale structure in the IMECOCAL region (off Baja California) from hydrographic, ADCP and altimetry data. Deep-Sea Research, Part II 51: 785-798.

Stouthamer CE \& MB Bain. 2012. Quantifying larval fish habitat in shoreline and shallow waters of the tidal Hudson River. Section VII. In: Fernald SH, DJ Yozzo \& H Andreyko (eds). Final reports of the Tibor T. Polgar Fellowship Program, 2010, pp. 1-25. Hudson River Foundation, New York.

Theilacker GH. 1978. Effect of starvation on the histological and morphological characteristics of jack mackerel, Trachurus symmetricus, larvae. Fishery Bulletin 76: 405414

Turner JT. 1984. The feeding ecology of some zooplankters that are important prey items of larval fish. NOAA Technical Report NMFS 7: 1-28.

Weber ED \& S McClatchie. 2012. Effect of environmental conditions on the distribution of Pacific mackerel (Scomber japonicus) larvae in the California Current system. Fishery Bulletin 110(1): 85-97.

Werner RG 2002. Habitat requirements. In: Fuiman LA \& RG Werner (eds). Fishery science, the unique contributions of early life stages, pp. 161-182. Blackwell Publishing. New York.

Westernhagen HV \& H Rosenthal. 1981. On condition factor measurements in Pacific herring larvae. Helgolaender Meeresuntersuchungen 34: 257-262.

Yúfera M, E Pascual, A Polo \& MC Sarasquete. 1993. Effect of starvation on the feeding ability of gilthead seabream (Sparus aurata L.) larvae at first feeding. Journal of Experimental Marine Biology and Ecology 169: 259-272. 